\title{
Optimal and Suboptimal Vibration Control of Structures
}

\author{
Mohammad S. Miah, Michael Kaliske \\ Institute of Structural Analysis, Faculty of Civil Engineering \\ Dresden University of Technology- TU Dresden \\ von Mises-Bau (VMB), Georg-Schumann-Str. 7, 01187 Dresden, Germany \\ Mohammad-Shamim.Miah@tu-dresden.de; michael.kaliske@ tu-dresden.de
}

\begin{abstract}
Structures subjected to extreme dynamic loads such as earthquake, impulse load always creates undesirable vibration. And the unexpected extreme deformation may lead to structural partial damage or fully collapse situation. Hence it is essential to understand the dynamical behaviour of structures subjected to those type of extreme loads. Typically, structures are combined with optimal control algorithms for vibration mitigation. And the desired control force is estimated based on the measured information e.g. displacement, velocity. To do this end, a 6-storied frame is coupled with an optimal control law known as the viscous damping with negative stiffness (VDNS). Additionally, a modified passive control technique is adopted namely the equivalent viscous damping (EVD) and the results are compared with the VDNS control scheme. The performance of the early mentioned strategies are evaluated numerically by employing different type of dynamic loads such as a real earthquake data (e.g. Loma Prieta 1989), a sinusoidal load and an irregular impulse load. A significant reduction of vibration is observed by optimally tuned control law as well as the sub-optimal performance of equivalent passive systems is presented.
\end{abstract}

Keywords: Earthquake, Impulse Load, Viscous Damping with Negative Stiffness, Optimal Control, Vibration Mitigation.

\section{Introduction}

The structures are vulnerable to the extreme dynamic loads such as seismic load, volcanic eruption, gale load and irregular or regular large impulse loads. It is often crucial to keep structures in safe condition due to extreme unexpected vibration generated by aforementioned loads. And modern structures even more complex as they are often coupled with different advanced technologies such as vibration mitigation mechanisms. Among them passive systems are one of the oldest technology used for supressing vibration where no sensors and electric power is needed [1]. However, due to the technological developments many smarter technologies than the passive systems have been introduced into the field of structural engineering over the last few decades. And those smart systems require control algorithms for operational purpose. The actuation principle is being used since long in the area of control engineering. Typically, the active control systems are a combination of sensors (to measure the quantities) and actuator (to generate force) and a control logic is necessary to estimate the control force based on the measured quantities [1][2] and [3].

The use of negative stiffness in the area of vibration mitigation and control is quite new and further development is needed. In existing literature, [4] presented different alternatives that can induce negative stiffness and the potentiality of it. Afterward, several researchers have used the concept and implemented for structural vibration control [5], [6] and [7]. Additionally, the effectiveness of the negative stiffness has reported by [7] and [8]. There are many control algorithms available for the vibration mitigation and control applications. Among them most commonly the following alternative can be found in the literature linear-quadratic-regulator (LQR) [9], viscous damping with negative stiffness [7], viscous damping, acceleration feedback [3]. The experimental validation of novel semi-active control approach with a rotational type magnetorheological damper is reported in [10].

The aforementioned algorithms required full-state or partial-state feedback for estimating the control force. Typically, full-state feedback may not be feasible as all of the degrees of freedom information are not measurable or not even realistic. Hence it is also, often desirable that less information is required by the control algorithm. However, the goal is to apply the optimal control so that the unexpected vibration is mitigated. In order to get the utmost advantage herein the application of negative stiffness is adopted. And the goal is achieved by employing the control law namely viscous damping with negative stiffness (VDNS). The performances of the VDNS is investigated by considering collocated 
(measured sensor and damper location is in the same location) information. And the numerical investigations are performed by using impulse type input excitations, a real seismic data of Loma Prieta 1989 and a harmonic type load. The active control principle is employed by considering a 6-storied frame. The early mentioned structure was model as 6 degrees of freedom (DOF) system. In order to evaluate the performance of the investigate scheme different aforementioned dynamic loads are employed and the performances are evaluated and compared. And an uncontrolled case was considered where no damper is used, meanwhile, another case is considered where damper was used. The VDNS control law is adopted herein for estimating the control force. Further, EVD is employed to represent an equivalent passive control system. It is assumed that all of the floors information are not measureable.

\section{Mathematical Problem Formulation}

In order to perform numerical investigations a benchmark model of a 6-DOF frame is considered where no damper is used. The aforementioned case will be called as uncontrolled, meanwhile, the case with dampers is named as controlled. It is essential to have a simple control principle to avoid instability of the implemented closed-loop. Hence the VDNS control law is adopted due to its optimal performance but very simple structure. The studied dynamical system is described below via the equation of motion.

$$
\underbrace{\left[\begin{array}{ccc}
m_{1} & \cdots & 0 \\
\vdots & \ddots & \vdots \\
0 & \cdots & m_{6}
\end{array}\right]}_{M} \ddot{X}(t)+\underbrace{\left[\begin{array}{ccc}
c_{1}+c_{2} & -c_{2} & \cdots \\
-c_{2} & \ddots & \vdots \\
\vdots & \cdots & c_{6}
\end{array}\right]}_{C} \dot{X}(t)+\underbrace{\left[\begin{array}{cccc}
k_{1}+k_{2} & -k_{2} & \cdots \\
-k_{2} & \ddots & \vdots \\
\vdots & \cdots & k_{6}
\end{array}\right]}_{K} X(t)=\gamma \times F(t)-M^{-1} \times f_{w}
$$

where $M$ is the mass matrix, $C$ indicates the damping matrix, $K$ means the stiffness matrix of the system, $X$ is the displacement vector, $\dot{X}$ and $\ddot{X}$ are the first and second time derivative of the displacement, $t$ represents the time vector, $\gamma$ is a control vector that controls the controller's location, $f_{w}$ is the exogenous input excitation.

It is mentioned earlier that the VDNS is an optimal control law but has very simple structure in contrary to LQR control law that requires full-state feedback. Typically, it is not feasible to place sensor in all of the DOF of the structures. That will be very costly solution and will also require high data acquisition as well as data storing systems. Hence it is always preferable to have something that is optimal but very simple to use. For the fulfilment of this study, the VDNS control law is briefly presented here. Nonetheless, interested readers are advised to get more information regarding VDNS via [7]-[8].

$$
f^{c t}(t)=-k^{c t} \times x(t)+c^{c t} \times \dot{x}(t)
$$

where $f^{c t}$ indicates the optimal control force based on the given input, $k^{c t}$ is the stiffness of the damper and $c^{c t}$ is the viscous damping coefficients of the controller. Be noted the minuses sign (-) before $k^{v d n s}$ that is to confirm the negative stiffness. The rest of the variable has the same meaning as before. However, it needs to be mentioned in case passive control system that is typically relies on stiffness. Hence for the equivalent viscous damping (EVD) it is assumed that damping coefficient $\left(c^{c t}\right)$ negligible because the stiffness is assumed to be the governing parameter. The state-space formulation is adopted to have a compact problem formulation instead of having several individual equation of motion of each degree-of-freedom. The early mentioned formulation has two main equations. The first one is known as the system equation that describe the dynamical systems. The system equation contain mass, stiffness, damping and input excitation information as well as controller's location.

$$
\dot{Z}(t)=\boldsymbol{A Z}(t)+\boldsymbol{B} u(t)
$$


where the system matrix represents by $A$, typically, contains mass, damping and stiffness information $A=$ $\left[\begin{array}{cc}0_{n \times n} & I_{n \times n} \\ -M^{-1} K & -M^{-1} C\end{array}\right], n$ means the degree of freedom, B means the input matrix, where the number of columns indicates whether the system has input force only or input force with damper, $Z$ indicates the state vector, usually, contains displacement and velocity vector, $u$ is the input force. And the second equation is called the measurement equation that basically describes all the quantities (e.g. displacement, velocity, acceleration) designers want to measure.

$$
Y(t)=\boldsymbol{H} Z(t)+\boldsymbol{D} u(t)
$$

where $H$ indicates the output matrix, that control the desired measurements such as displacement, velocity and accelerations and $D$ is the feedthrough matrix.

\section{Numerical Implementations}

The efficacy of the studied approach is preliminarily investigated numerically. The numerical investigation is performed via MATLAB ${ }^{\circledR}$ and SIMULINK $^{\circledR}$. A reference model is considered where no control approach was implemented, namely "uncontrolled" case. While the structure coupled with virtual damper is named as "controlled". Henceforth, for the simplicity, the aforementioned named of the cases will be used to explain the results. The studied closed-loop is shown in Figure 1.

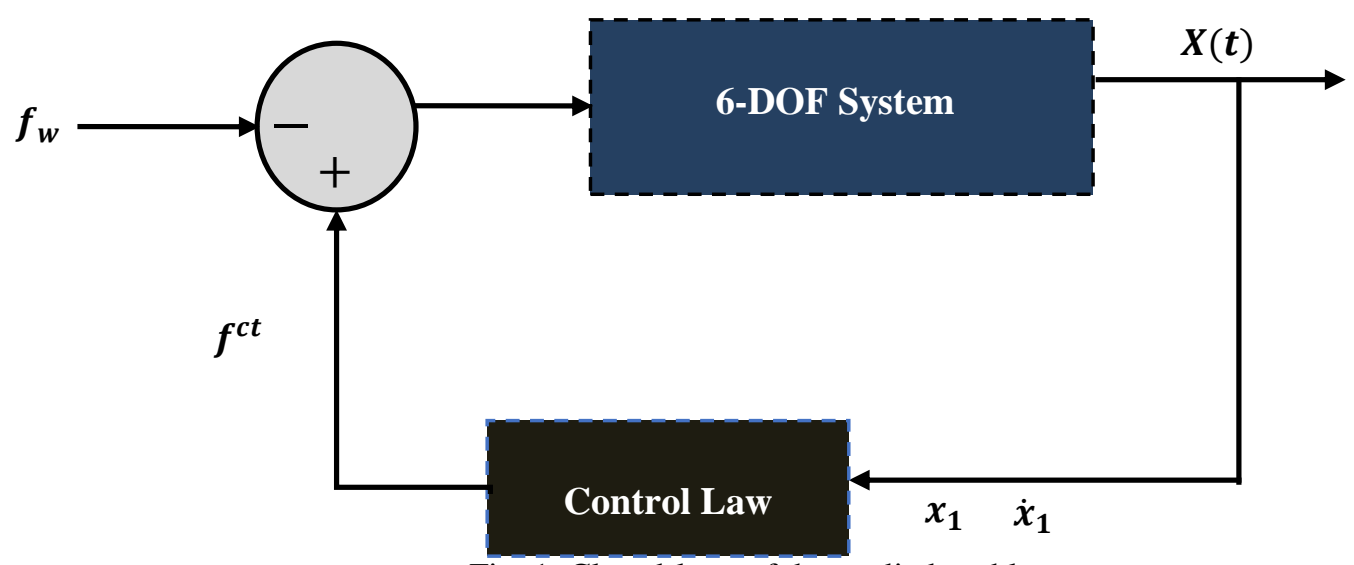

Fig. 1: Closed-loop of the studied problem.

Herein three different types of dynamic loads such as; (i) irregular impulse/impact, (ii) Loma Prieta 1989 earthquake data and (iii) harmonic loads are employed and depicted in Figure 2. The irregular impulse load that has an impact period of $1 \mathrm{sec}$ is generated for the implementations. The Loma Prieta 1989 seismic data is also employed to evaluate the dynamic response of the considered problem. Additionally, a sinusoidal load is used as input excitation such that the load was applied at the first critical frequency of the structure. In order to suppress structural movement due to the external excitations two dampers are used where the first one was placed at first floor level and the second one is at sixth floor level.

Firstly, the results such as top floor's displacement and velocity are presented herein in Figures 3 and 4 respectively for impulse load. The colour coding for the aforementioned figures remain same. Where the green colour represents the uncontrolled case, the red solid line indicates controlled case with VDNS and the black solid line means the controlled case with EVD. In order to evaluate the response of the structure during the shock and after the shock is considered. Hence even though the impact load duration is only $1 \mathrm{sec}$ but the response was recorded until $\pm 30 \mathrm{secs}$. It is noticeable that such extreme impact load definitely will create serious damage, however, the optimally tuned VDNS shows significant 
reduction of vibration. Nevertheless, in case of EVD it is visible that the control approach is sub-optimal and the overall response are not reduced such case is comparable with passive control approach. It needs to be mentioned that the controller's parameters remain same for all of the dynamic loads.
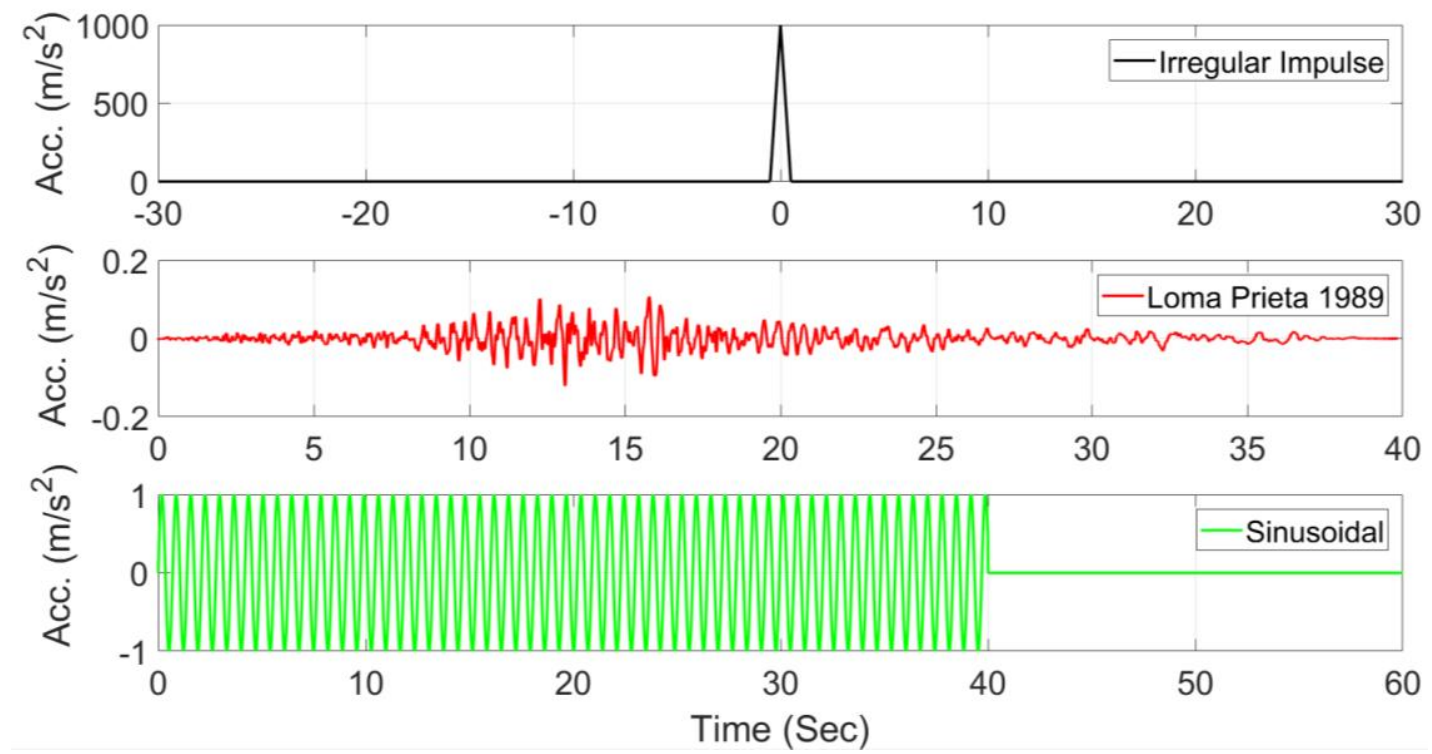

Fig. 2: The input excitations.
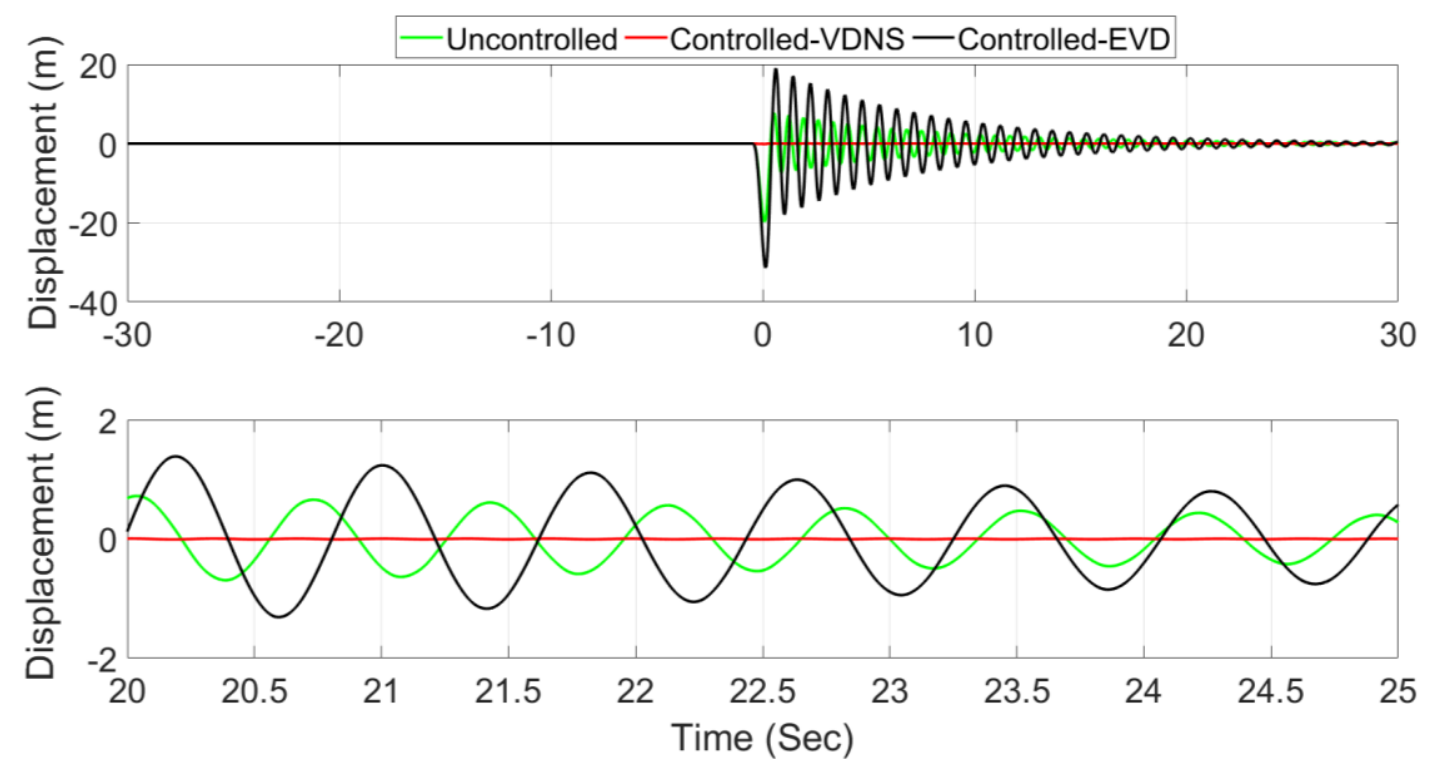

Fig. 3: Top floor's displacement of the frame under irregular impulse input. 

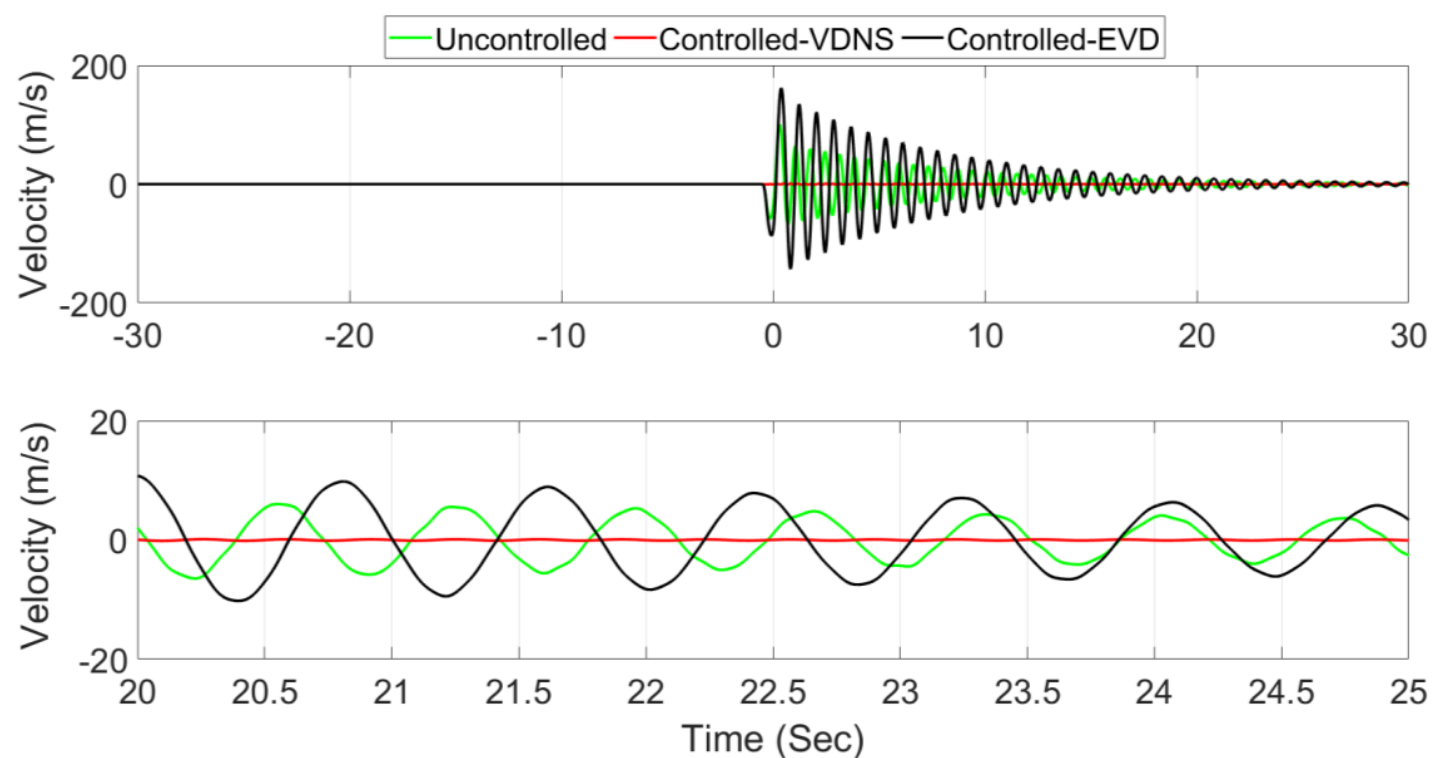

Fig. 4: The $6^{\text {th }}$ floor velocity of the frame under irregular impulse input.

In order to verify the early statement the Loma Prieta 1989 earthquake data is employed and the response are evaluated and compared. The results of top floor such as $6^{\text {th }}$ floor's displacement and velocity are displayed in Figures 5 and 6 accordingly. The colour coding for the aforementioned figures remain same as before (e.g. Fig. 3 and 4). Interestingly, in this case also the optimally tuned VDNS shows superior performance than EVD. And it is also evident that the EVD control approach is sub-optimal.
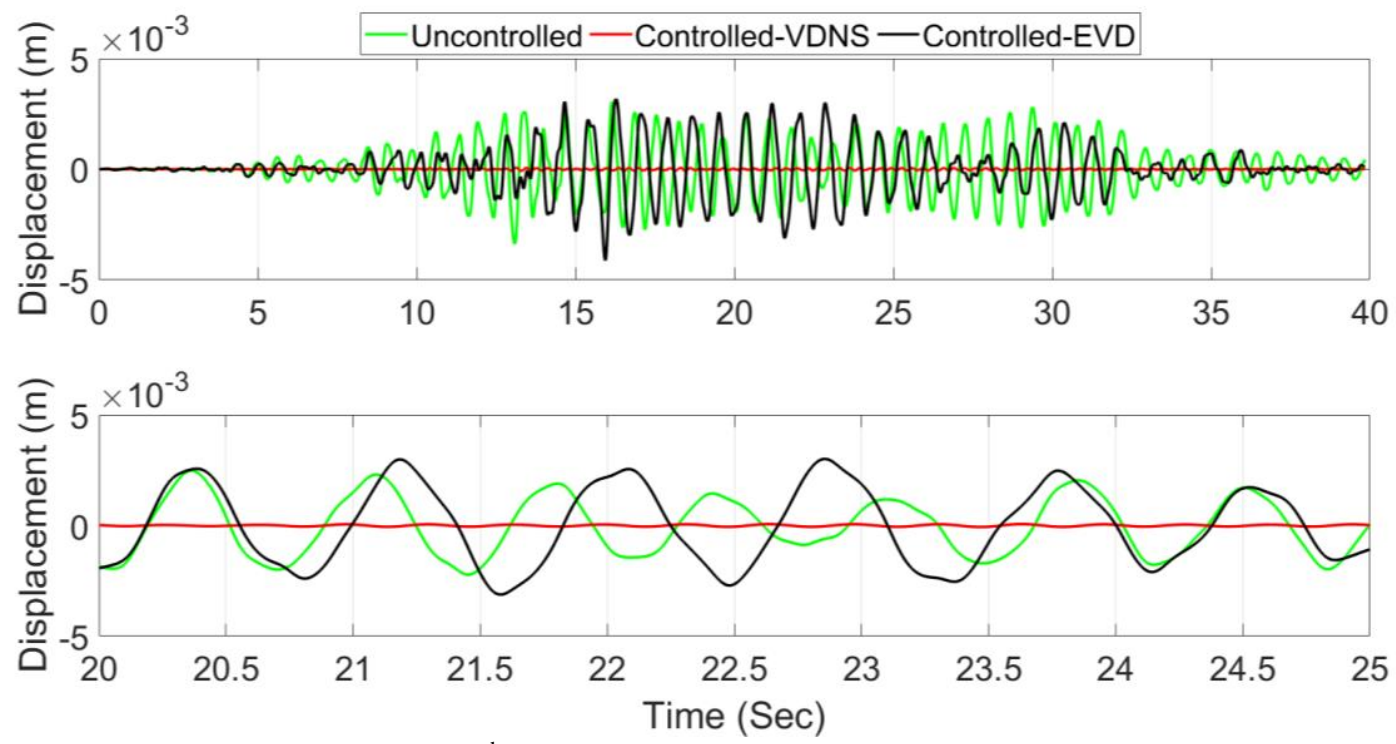

Fig. 5: The $6^{\text {th }}$ floor displacement due to earthquake load. 

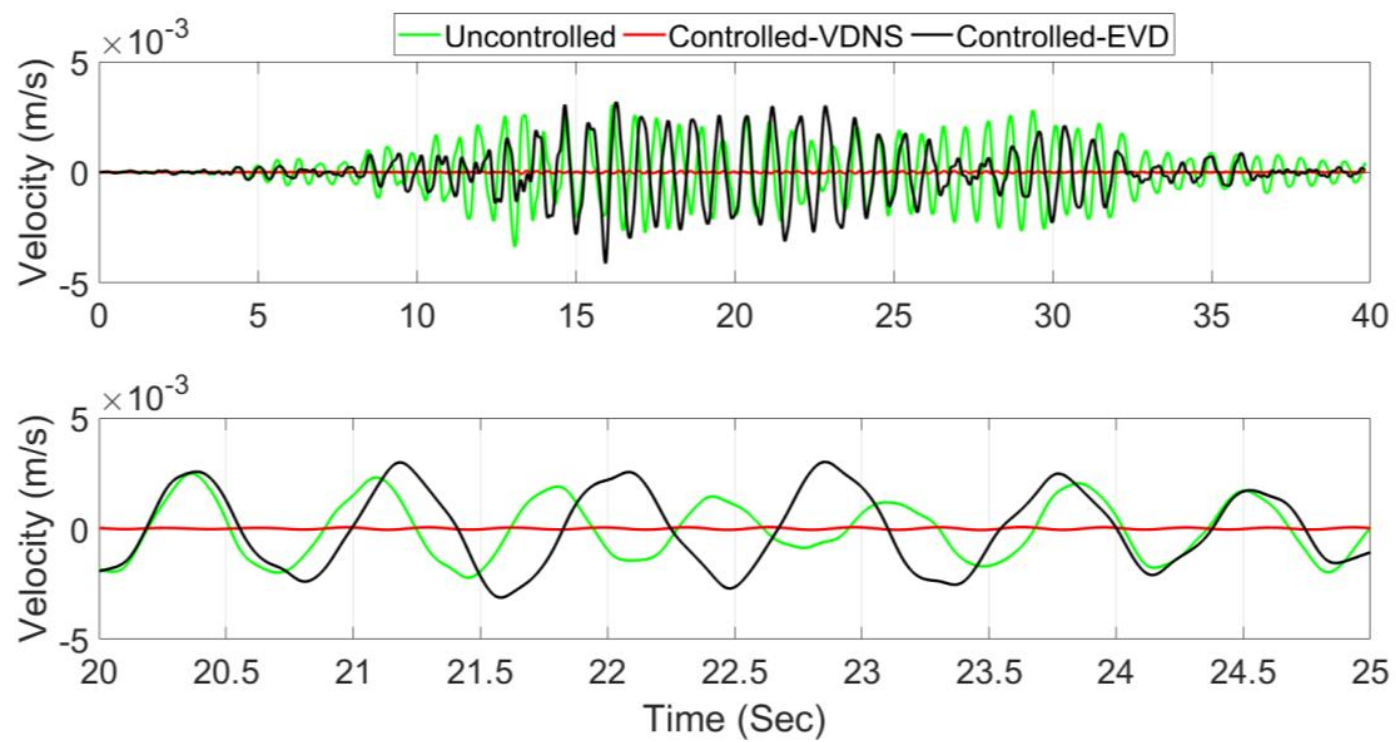

Fig. 6: The $6^{\text {th }}$ floor velocity due to earthquake load.

Further, a harmonic type load is also employed to verify the performance of the studied approach. In this case the $6^{\text {th }}$ floor's displacement is presented in Figure 7. Meanwhile, Figure 8 shows the comparison of the velocity of the same floor. And the colour coding remains same as before and the optimally tuned VDNS better performance than EVD. It can be sated that the optimally tuned controller may performed better even though the loads are totally different both in their magnitudes and excitation frequency characteristics.
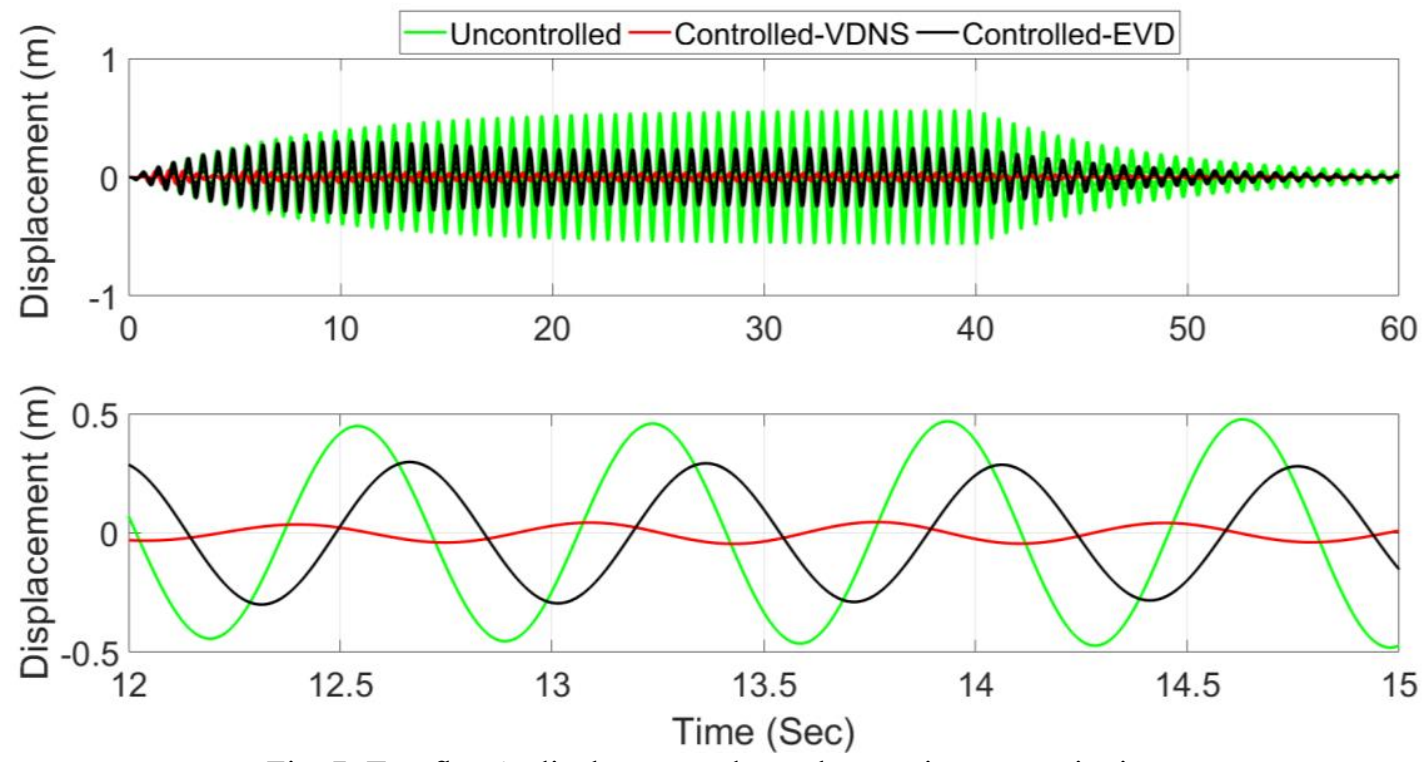

Fig. 7: Top floor's displacement due to harmonic type excitation. 

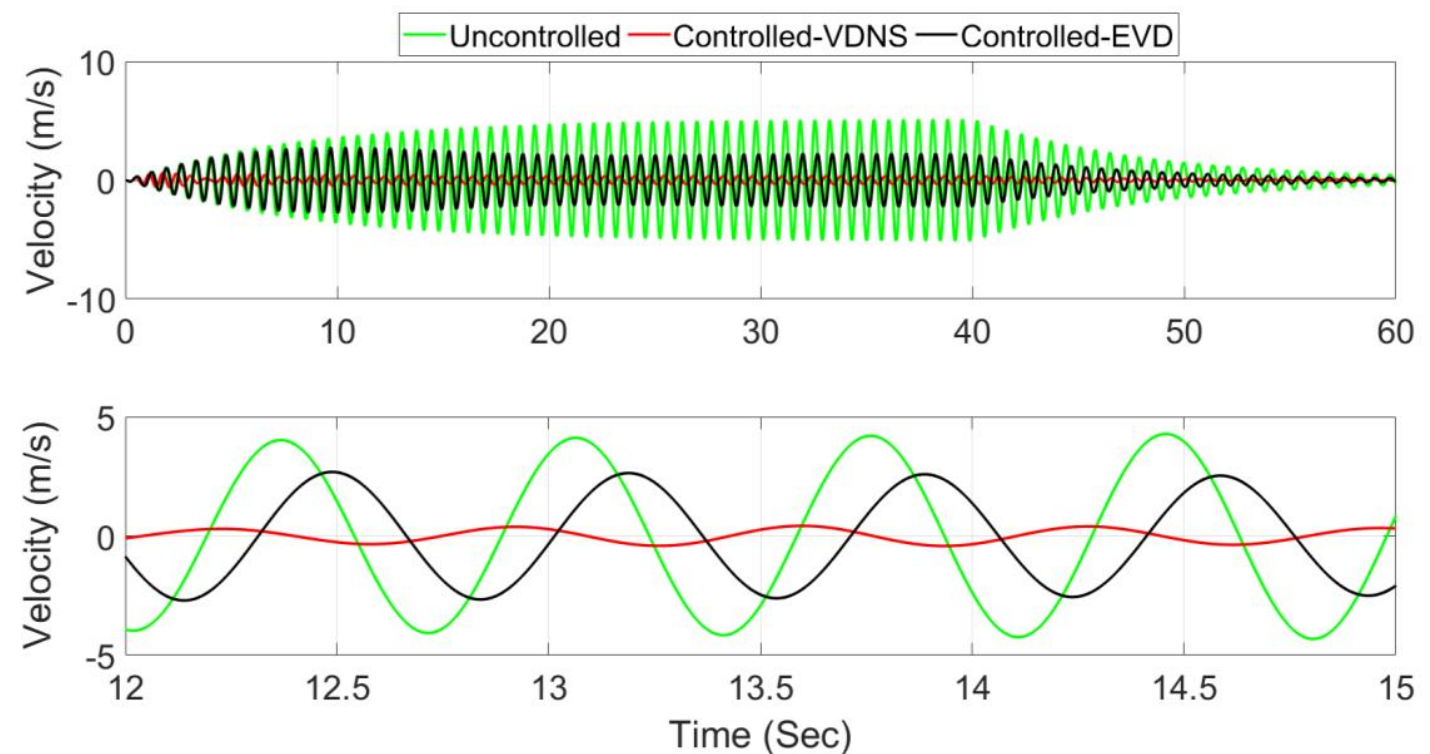

Fig. 8: Top floor's velocity of the structure subjected to harmonic type excitation.

Finally, in order to see the performance of the studied approach in numbers the peak values are summarized in Table 1. Where it can be found that the controller has significantly reduced the peak values for all of the extreme dynamic loads.

Table 1: Summary of peak amplitudes.

\begin{tabular}{|c|c|c|c|c|}
\hline \multirow{2}{*}{ Type of Excitations } & \multicolumn{2}{|c|}{ Maximum $(\mathrm{m})$} & \multicolumn{2}{c|}{ Minimum $(\mathrm{m})$} \\
\cline { 2 - 5 } & Uncontrolled & Controlled & Uncontrolled & Controlled \\
\hline Sinusoidal & 0.5692 & 0.0661 & -05691 & -0.644 \\
\hline Irregular Impulse & 7.7574 & 0.0652 & -19.8038 & -0.1284 \\
\hline Loma Prieta 1989 & 0.0031 & 0.0008223 & -0.0034 & -0.0008374 \\
\hline
\end{tabular}

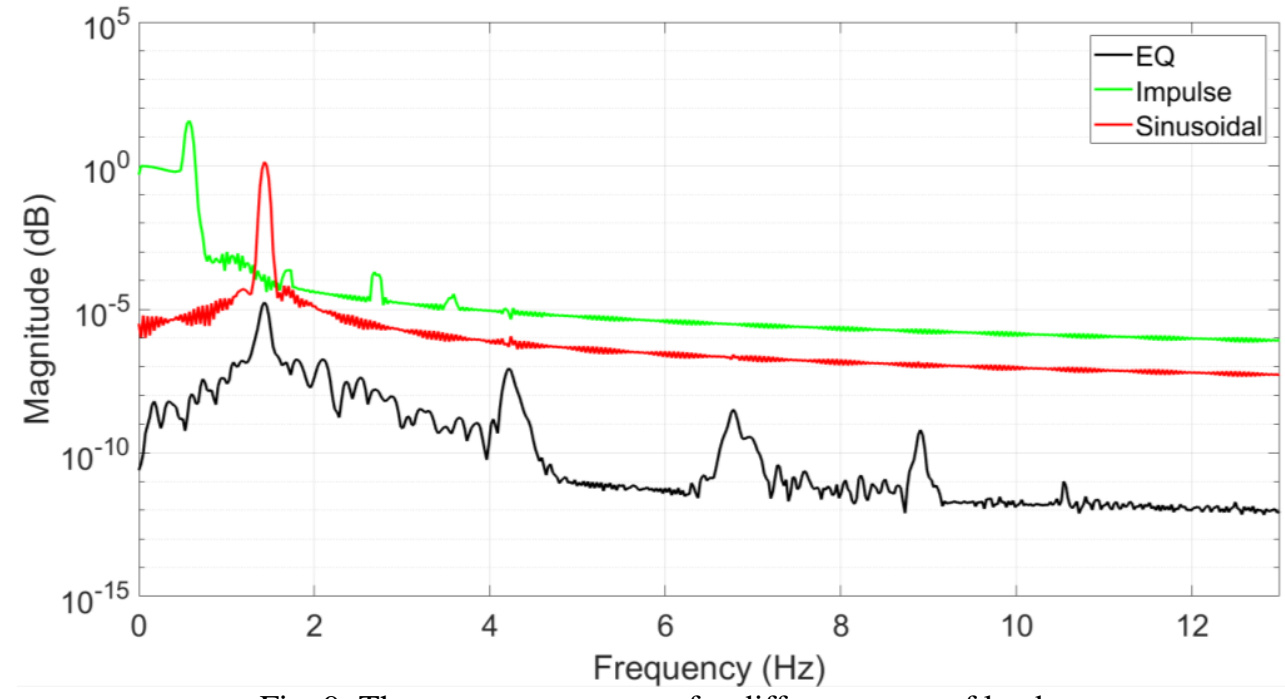

Fig. 9: The response spectrum for different types of load. 
The spectrum of the structure under different loads are evaluated and it is observed that almost all of the resonant frequencies are visible. However, higher frequencies are filtered out to visualize desired frequencies. The spectrums are presented in Figure 9, and it is visible that the modes under earthquake load was excited more than other loads. This is due to the frequency content of the excitation signals.

\section{Conclusion}

This study investigates the vibration mitigation of a 6-storeid structure by employing the Loma Prieta 1989 earthquake, harmonic load and impulse load. Two dampers are used to reduce not only the first mode vibration but also other modes. More specifically, two dampers are employed at 1st floor level and 6th floor level accordingly. In order to evaluate the response of the studied problem a benchmark case is consider where no damper is considered referred as uncontrolled. Further, the aforementioned case is coupled with VDNS and EVD control laws namely controlled-VDNS and controlled-EVD respectively. It is observed that the implemented methodology is quite effective to reduce the extreme vibration quite efficiently for all of the dynamic loads. It needs to be mentioned that the controller properties remain unchanged for all of the loading cases. This study reveals that more than one dampers can be beneficial for the significant reduction of vibration. Last but not least, VDNS shows the superior performances than EVD in terms of overall reduction of unexpected amplitudes of the dynamical responses.

\section{Acknowledgements}

The authors acknowledge the grant number F-003661-Ü1G-1140901 of TU Dresden's Institutional Strategy, Dresden University of Technology-TU Dresden. Also would like to appreciate the support of the Institute of Structural Analysis, Faculty of Civil Engineering, Dresden University of Technology-TU Dresden, Dresden, Germany.

\section{References}

[1] S. J. Han, P. Tsopelas, and A. Baz, “Active/passive seismic control of structures," Journal of Earthquake Engineering, vol. 10, no. 4, pp. 509-526, 2006.

[2] A. Preumont, "Vibration control of active structures: an introduction," 2nd ed, Kluwer Academic Publishers, Dordrecht: Kluwer, The Netherlands, 2004.

[3] S. Dyke, "Acceleration feedback control strategies for active and semi-active control systems: modeling, algorithm development, and experimental verification," Notre Dame, Indiana: University of Notre Dame, 1996.

[4] D. L. Platus, "Negative-stiffness-mechanism vibration isolation systems," Proc. SPIE 1619, Vibration Control in Microelectronics, Optics, and Metrology, 1992. doi: 10.1117/12.56823.

[5] M. S. Miah, "Semi-active control for magnetorheological dampers via coupling of system identification methods," ETH-Zürich, Zurich, Switzerland, 22776, 2015.

[6] S. Bhowmik, "Modelling and Control of Magnetorheological Damper: Real-time implementation and experimental verification," Lyngby, Denmark: Technical University of Denmark, 2011.

[7] F. Weber and C. Boston, "Clipped viscous damping with negative stiffness for semi-active cable damping," Smart Materials and Structures, vol. 20, no. 4, pp. 1-13, 2011.

[8] M. S. Miah, E. N. Chatzi, and F. Weber, "MR Damper based Vibration Mitigation using a Bouc-Wen Model and a Nonlinear Observer," Sixth World Conference on Structural Control and Monitoring, pp. 1185-1194, 2014.

[9] B. D. O. Anderson and J. B. Moore, "Optimal Control Linear Quadratic Methods," Englewood Cliffs, Prentice-Hall, NJ: USA, 1989.

[10] M. S. Miah, E. N. Chatzi, V. K. Dertimanis and F. Weber, "Real-time experimental validation of a novel semi-active control scheme for vibration mitigation," Structural. Control Health Monitoring, vol. 24, pp. e1878, 2017. 